\title{
Terapia de Recanalização Endovascular de Oclusão da Artéria Carótida em Paciente com Acidente Vascular Cerebral Agudo
}

\author{
Andrés G. Sánchez ${ }^{1}$, Antonio M. Kambara ${ }^{1}$, Samuel M. Moreira ${ }^{1}$, Áurea J. Chaves ${ }^{1}$, Manuel N. Cano ${ }^{1}$, \\ Luiz A. Mattos ${ }^{1}$, Alexandre Abizaid', Fausto Feres' ${ }^{1}$, Amanda G.M.R. Sousa ${ }^{1}$, J. Eduardo Sousa ${ }^{1}$
}

\section{RESUMO}

Relatamos o caso de uma paciente de 69 anos, que, após a realização de arteriografia carotídea, apresentou trombose sintomática da bifurcação carotídea. A paciente foi submetida imediatamente a reperfusão endovascular mecânica, com implante bem-sucedido de stent carotídeo, obtendo reversão completa do déficit neurológico.

DESCRITORES: Acidente cerebrovascular. Embolia intracraniana. Reperfusão. Lesões das artérias carótidas, terapia.

\section{SUMMARY}

Percutaneous Recanalization of Carotid Artery Occlusion in Patients with an Acute Stroke

A 69 year-old woman developed symptomatic internal carotid artery thrombosis after carotid angiography. She was immediately submitted to mechanical endovascular reperfusion with successful carotid stent implantation and complete neurological recovery.

DESCRIPTORS: Cerebrovascular accident. Intracranial embolism. Reperfusion. Carotid artery injuries, therapy.
A trombose carotídea é uma das causas de acidente vascular cerebral (AVC) e pode ocorrer durante ou após a realização de angiografia cerebral diagnóstica ou de angioplastia carotídea. Diante dessa situação, há necessidade de terapia de reperfusão endovascular, a qual deve ser idealmente instituída o mais rápido possível, para que se obtenham resultados clínicos satisfatórios ${ }^{1}$.

\section{RELATO DO CASO}

Paciente I.S., do sexo feminino, com 69 anos de idade e história pregressa de hipertensão arterial sistêmica, dislipidemia, diabetes melito, angina instável e estenose assintomática das artérias carótidas internas, diagnosticada por ultra-som Doppler.

A paciente foi submetida a coronariografia e arteriografia dos vasos supra-aórticos, com a finalidade de confirmar a extensão das lesões coronarianas e caro-

\footnotetext{
1 Instituto Dante Pazzanese de Cardiologia - São Paulo, SP. Correspondência: Andrés Gustavo Sánchez Esteva. Alameda Franca, 386/12 - Jardim Paulista - São Paulo, SP - CEP 01422-000. E-mail: asanchez@bol.com.br

Recebido em: 28/9/2007 • Aceito em: 15/1/2008
}

tídeas, respectivamente. O exame físico inicial da paciente era normal, incluindo o exame neurológico.

A coronariografia foi realizada inicialmente e mostrou doença de múltiplos vasos. Durante a angiografia dos vasos cerebrais, foram canuladas de maneira seletiva e estudadas as artérias vertebrais e as artérias carótidas sem intercorrências. De acordo com o critério do North American Symptomatic Carotid Endarterectomy Trial (NASCET) ${ }^{2}$, a paciente apresentava estenose de $60 \%$ na artéria carótida interna direita e de $95 \%$ na artéria carótida interna esquerda.

Aproximadamente 10 minutos após a finalização do procedimento diagnóstico, a paciente apresentou afasia e hemiplegia direita. Em decorrência da alta probabilidade de trombose ou embolia arterial no território da artéria carótida interna esquerda, optou-se por repetir a angiografia carotídea esquerda (usando um cateter diagnóstico Simmons 5F), com estudo do segmento cervical e da circulação intracerebral correspondente, sendo observada oclusão da carótida comum esquerda (Figura 1).

Por causa da gravidade do quadro clínico, optouse pela tentativa de recanalização da artéria carótida esquerda. $O$ cateter diagnóstico foi trocado por um cateter-guia Multi-Purpose 8F, usando um guia 0,035" 
Sánchez AG, et al. Terapia de Recanalização Endovascular de Oclusão da Artéria Carótida em Paciente com Acidente Vascular Cerebral Agudo. Rev Bras Cardiol Invas. 2008;16(1):102-105.

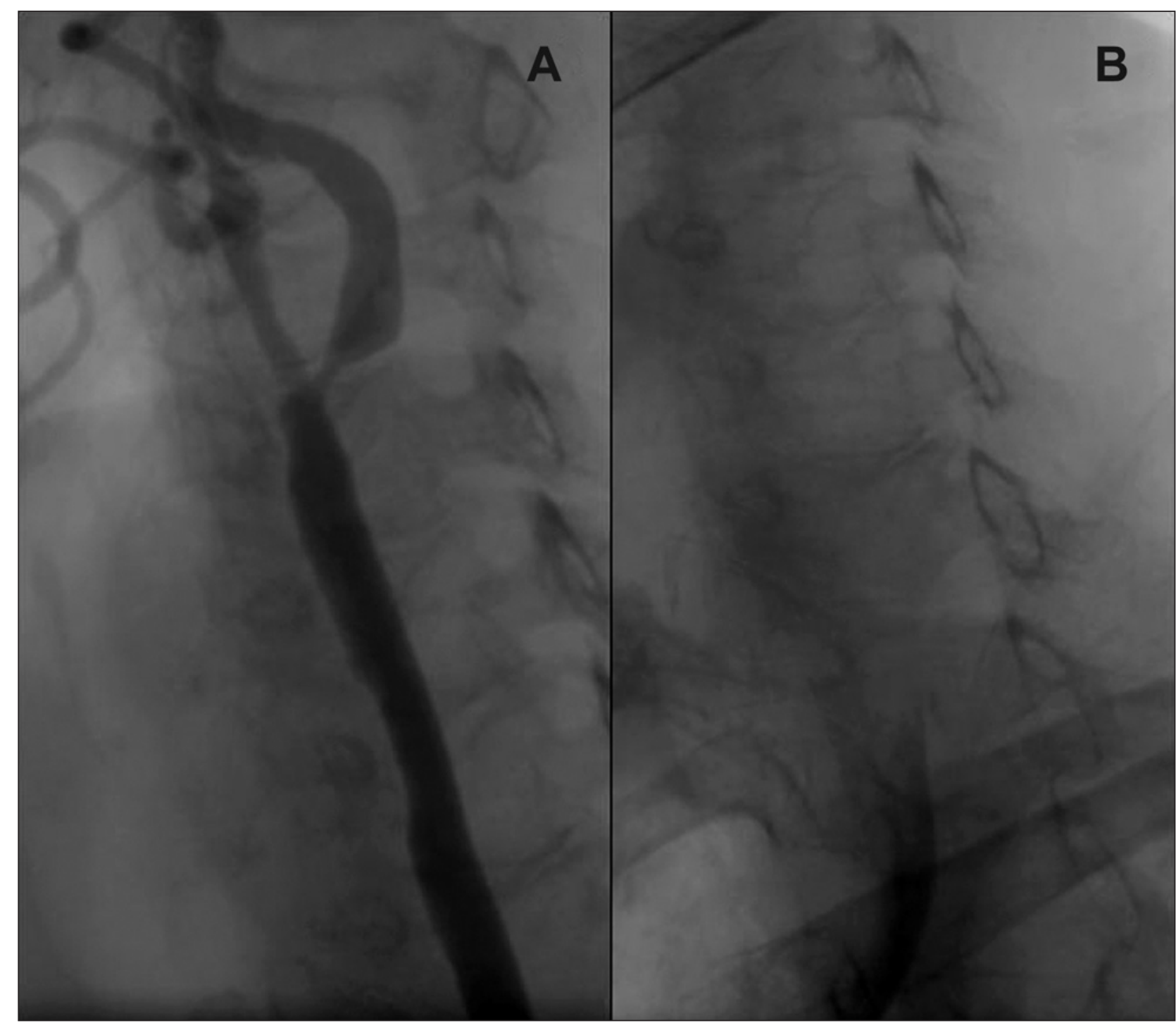

Figura 1 - Angiografia da artéria carótida interna esquerda. A: Durante o procedimento diagnóstico. B: No reestudo, 20 minutos após o acidente vascular cerebral agudo.

rígido, de $260 \mathrm{~cm}$ de comprimento. Subseqüentemente, foram iniciadas antiagregação plaquetária com 200 mg de ácido acetilsalicílico e 300 mg de clopidogrel por via oral, assim como anticoagulação com heparina não-fracionada endovenosa, na dose de $70 \mathrm{Ul} / \mathrm{kg}$. A paciente também recebeu tirofiban (Agrastat), por via endovenosa, em dose de ataque $(10 \mathrm{mcg} / \mathrm{kg})$ e, posteriormente, em dose de manutenção $(0,15 \mathrm{mcg} / \mathrm{kg} / \mathrm{min})$ por 12 horas.

A obstrução foi ultrapassada com guia medium support 0,014" de $180 \mathrm{~cm}$ de comprimento, tendo sido colocado um filtro de proteção distal através do guia. Pré-dilatação com balão 2,5 x 20 mm foi realizada, com restauração do fluxo após a terceira dilatação. A seguir, foi colocado um stent auto-expansível de nitinol do tipo Precise de 7,0 x $40 \mathrm{~mm}$ no local, realizando-se pós-dilatação (intra-stent) com balão $6,0 \times 20 \mathrm{~mm}$, diminuindo a lesão residual para 14\%. Imediatamente após a desobstrução, a paciente apresentou recuperação progressiva do déficit motor e da afasia.

A seguir, realizamos injeção de controle, obtendo-se desobstrução e fluxo normal nas artérias carótida comum e interna esquerda, com circulação e fluxo intracerebral normais (Figura 2) e reversão completa do déficit motor nas primeiras 24 horas. A tomografia computadorizada de crânio realizada dois dias após o procedimento foi normal (Figura 3).

Foi indicado tratamento com clopidogrel $(75 \mathrm{mg} /$ dia) por 30 dias após o procedimento e ácido acetilsalicílico (200 mg/dia) de forma indefinida. 
Sánchez AG, et al. Terapia de Recanalização Endovascular de Oclusão da Artéria Carótida em Paciente com Acidente Vascular Cerebral Agudo. Rev Bras Cardiol Invas. 2008;16(1):102-105.

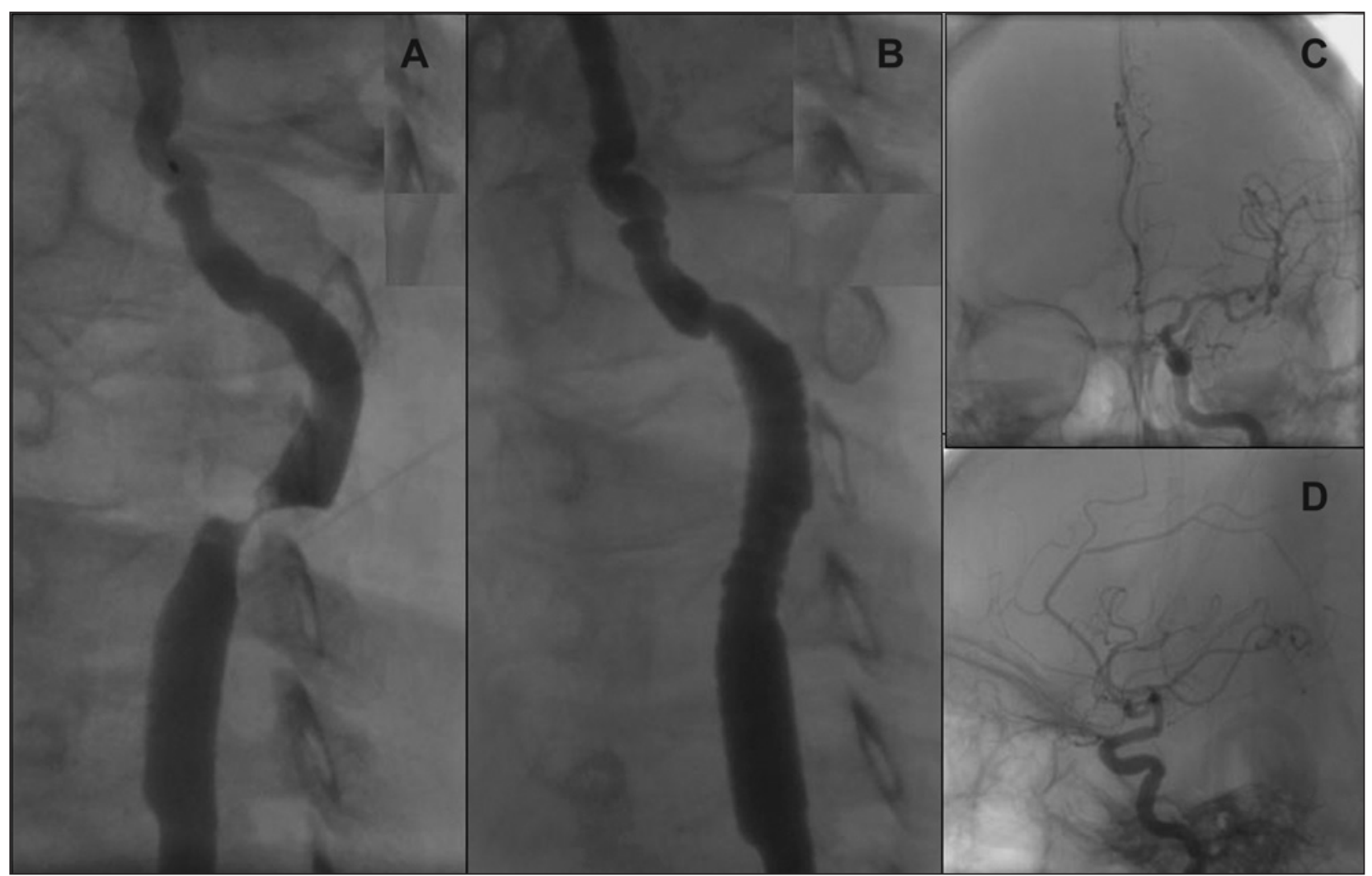

Figura 2 - Terapêutica de reperfusão endovascular. A: Após colocação do filtro e pré-dilatação. B, C e D: Controle final (do segmento cervical e intracerebral).

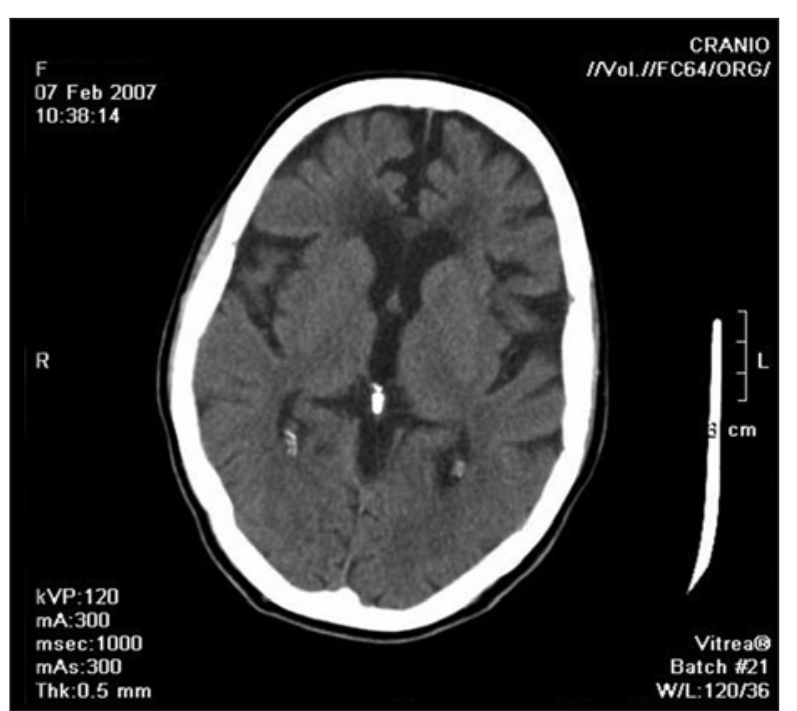

Figura 3 - Tomografia computadorizada de crânio, nas 48 horas após terapia de reperfusão endovascular no acidente vascular cerebral agudo.

\section{DISCUSSÃO}

O AVC secundário a trombose e/ou embolia cerebral é uma complicação pouco freqüente da angiografia cerebral, porém com elevada morbidade e mor- talidade ${ }^{3}$. O tratamento clínico é, na maioria dos casos, a modalidade escolhida, porém os resultados são insatisfatórios.

Na última década, surgiram novas modalidades terapêuticas na tentativa de minimizar as seqüelas dessa complicação, principalmente nas primeiras seis horas após o evento neurológico agudo ${ }^{1}$. As opções de terapia de reperfusão endovascular descritas na literatura são: uso de agentes fibrinolíticos endovenosos ${ }^{4,5}$, intra-arteriais ${ }^{6}$ ou combinados ${ }^{7,8}$, realização de aspiração de trombo $0^{9,10}$ ou intervenção endovascular percutânea ${ }^{11}$, associados ou não à realização de inibidores da glicoproteína $\mathrm{Ilb} / \mathrm{IIla}^{12-14}$.

Assim, com base em nossa experiência positiva crescente ${ }^{15}$, concluímos que o estudo dos vasos supra-aórticos nos segmentos cervical e intracerebral deve ser feito após procedimentos endovasculares em pacientes com suspeita de AVC e que a terapia de reperfusão endovascular imediata, associada ou não à infusão dos inibidores da glicoproteína IIb/IIla, deva ser considerada medida terapêutica importante em pacientes com trombose carotídea aguda.

\section{REFERÊNCIAS BIBLIOGRÁFICAS}

1. Rha JH, Saver JL. The impact of recanalization on ischemic stroke outcome. a meta-analysis. Stroke. 2007;38(3):967-73. 
Sánchez AG, et al. Terapia de Recanalização Endovascular de Oclusão da Artéria Carótida em Paciente com Acidente Vascular Cerebral Agudo. Rev Bras Cardiol Invas. 2008;16(1):102-105.

2. North American Symptomatic Carotid Endarterectomy Trial (NASCET) Collaborators. Beneficial effect of carotid endarterectomy in symptomatic patients with high-grade carotid stenosis. N Engl J Med. 1991;325(7):445-53.

3. Heiserman JE, Dean BL, Hodak JA, Flom RA, Bird CR, Drayer BP, et al. Neurologic complications of cerebral angiography. AJNR Am J Neuroradiol. 1994;15(8):1401-11.

4. Ribo M, Molina CA, Rovira A, Quintana M, Delgado P, Montaner J, et al. Safety and efficacy of intravenous tissue plasminogen activator stroke treatment in the 3- to 6-hour window using multimodal transcranial Doppler/MRI selection protocol. Stroke. 2005;36(3):602-6.

5. Kent DM, Ruthazer R, Selker HP. Are some patients likely to benefit from recombinant tissue-type plasminogen activator for acute ischemic stroke even beyond 3 hours from symptom onset? Stroke. 2003;34(2):464-7.

6. Furlan A, Higashida R, Wechsler L, Gent M, Rowley $H$, Kase $C$, et al. Intra-arterial prourokinase for acute ischemic stroke. The PROACT II study: a randomized controlled trial. Prolyse in Acute Cerebral Thromboembolism. JAMA. 1999;282(21):2003-11.

7. Lewandowski CA, Frankel M, Tomsick TA, Broderick J, Frey J, Clark W, et al. Combined intravenous and intra-arterial r-TPA versus intra-arterial therapy of acute ischemic stroke: Emergency Management of Stroke (EMS) Bridging Trial. Stroke. 1999;30(12):2598-605.

8. IMS Study Investigators. Combined intravenous and intra- arterial recanalization for acute ischemic stroke: the Intervention Management of Stroke Study. Stroke. 2004;35(4):904-11.

9. Lutsep HL, Clark WM, Nesbit GM, Kuether TA, Barnwell SL. Intraarterial suction thrombectomy in acute stroke. AJNR Am J Neuroradiol. 2002;23(5):783-6.

10. Xu GF, Suh DC, Choi CG, Kim JK, Kim W, Kim SJ, et al. Aspiration thrombectomy of acute complete carotid bulb occlusion. J Vasc Interv Radiol. 2005;16(4):539-42.

11. Sugg RM, Malkoff MD, Noser EA, Shaltoni HM, Weir R, Cacayorin ED, et al. Endovascular recanalization of internal carotid artery occlusion in acute ischemic stroke. AJNR Am J Neuroradiol. 2005;26(10):2591-4.

12. Straub S, Junghans U, Jovanovic V, Wittsack HJ, Seitz RJ, Siebler M. Systemic thrombolysis with recombinant tissue plasminogen activator and tirofiban in acute middle cerebral artery occlusion. Stroke. 2004;35(3):705-9.

13. Lee DH, Jo KD, Kim HG, Choi SJ, Jung SM, Ryu DS, et al. Local intraarterial urokinase thrombolysis of acute ischemic stroke with or without intravenous abciximab: a pilot study. J Vasc Interv Radiol. 2002;13(8):769-74.

14. Cloft HJ, Samuels OB, Tong FC, Dion JE. Use of abciximab for mediation of thromboembolic complication of endovascular therapy. AJNR Am J Neuroradiol. 2001;22(9):1764-7.

15. Sánchez AG, Kambara AM, Moreira SM, Franchetti M, Cano $M N$, Feres $F$, et al. Reperfusão endovascular pós-embolia cerebral durante arteriografia carotídea. Rev Bras Cardiol Invas. 2006;14(2):178-80. 